\title{
SMOOTH SOLUTIONS OF VOLTERRA EQUATIONS VIA SEMIGROUPS
}

\author{
TOMÁŠ BÁRTA
}

(Received 18 December 2007)

\begin{abstract}
In this paper we introduce a class of left shift semigroups that are differentiable. With the help of perturbation theory for differentiable semigroups we show that solutions of an integrodifferential equation can be infinitely differentiable if the convolution kernel is sufficiently smooth and regular.
\end{abstract}

1991 Mathematics subject classification: primary 47D06; secondary 45D05.

Keywords and phrases: differentiable semigroup, bounded perturbation, shift semigroup, integrodifferential equation.

\section{Introduction}

In this paper, we are interested in the semigroup approach to the following integrodifferential equation

$$
\begin{aligned}
& \dot{u}(t)=A u(t)+\int_{0}^{t} B(t-s) u(s) d s, \\
& u(0)=x,
\end{aligned}
$$

where $A$ is the generator of a $C_{0}$-semigroup on a Banach space $X$ and $B: \mathbb{R}_{+}$ $\rightarrow B(D(A), X)$. It was the main objection against the semigroup approach for many years that it is not possible to obtain regularity of the solutions. This is not true. In [1] we have shown via semigroups that the solutions of (1.1) are analytic provided the semigroup generated by $A$ is analytic and $B$ and $B^{\prime}$ have analytic extensions to a sector

$$
\Sigma_{\varphi}:=\{z \in \mathbb{C}: z \neq 0 \text { and }|\arg z| \leq \varphi\} .
$$

In [2] we have shown maximal $L_{p}$-regularity for (1.1) via semigroups. In the present paper, we show that solutions of (1.1) are $C^{\infty}$ if $A$ generates a differentiable semigroup

This work has been created as a research activity of Nečas Center for Mathematical Modeling LC 06052 (MŠMT ČR), partly supported also by the grants 201/06/P171 (GA ČR), and MSM 21620839 (MŠMT CR).

(C) 2008 Australian Mathematical Society 0004-9727/08 \$A2.00+0.00 
and the kernel $B$ has an analytic extension to a set of an appropriate shape. This result on differentiability is neither stronger nor weaker than the result obtained by Prüss (see [10, Theorems 3.1 and 7.2]). Comparison of the two results can be found at the end of this paper.

The solutions of (1.1) are given by the first coordinate of the semigroup $\mathcal{T}$ generated by

$$
\mathcal{A}=\left(\begin{array}{cc}
A & \delta_{0} \\
B(\cdot) & D
\end{array}\right), \quad D(\mathcal{A})=D(A) \times D(D)
$$

if $\mathcal{A}$ is a generator. Here $\delta_{0} f:=f(0)$ is the Dirac operator and $D f:=f^{\prime}$ is the first derivative. Properties of $\mathcal{T}$ depend of course on $A$ but also on the space on which acts the operator $D$. So, we shall start in Section 2 with the definition of spaces $Y^{q}$, $Y^{\infty}$, where the operator $D$ defines a differentiable translation semigroup. Then we use perturbation theory. It is obvious that the operator

$$
\mathcal{A}_{00}:=\left(\begin{array}{cc}
A & 0 \\
0 & D
\end{array}\right) \quad \text { generates } \mathcal{T}_{00}(t)=\left(\begin{array}{cc}
T(t) & 0 \\
0 & S(T)
\end{array}\right),
$$

which is differentiable if $T$ and $S$ are differentiable. We perturb $\mathcal{A}_{00}$ by

$$
\left(\begin{array}{cc}
0 & \delta_{0} \\
0 & 0
\end{array}\right) \text { and }\left(\begin{array}{cc}
0 & 0 \\
B(\cdot) & 0
\end{array}\right)
$$

respectively and show that also the perturbed operator $\mathcal{A}$ generates a differentiable semigroup. Section 3 is therefore devoted to the perturbation theory for differentiable semigroups. It contains results that are applied in Section 4, but some of them may be interesting on their own. In Section 4 we obtain $C^{\infty}$-solutions of (1.1). The main results of this paper are Theorems 4.1 and 4.2. Finally we compare these results to [10, Theorems 3.1 and 7.2].

\section{Differentiable translation semigroups}

In [1] we have shown that the left translation semigroup on the space of bounded holomorphic functions on a sector, and also on the space of holomorphic functions on a sector that are $L^{p}$-integrable, is analytic. In this section we introduce translation semigroups on spaces of holomorphic functions defined on other areas.

Definition 2.1. We say that a function $h: \mathbb{R}_{+} \rightarrow \mathbb{R}_{+}$is admissible if $h$ is increasing and convex and $h(0)=0$. For an admissible function $h$ we define

$$
\Sigma_{h}:=\{x+i y \in \mathbb{C}: x>0 \text { and }|y|<h(x)\} .
$$

By $\varphi_{h}$ let us denote the supremum of all angles of sectors contained in $\Sigma_{h}$, that is, $\varphi_{h}:=\sup \left\{\operatorname{arctg} h^{\prime}(x): x \in \mathbb{R}_{+}\right\}$. 
Let us define the spaces

$$
Y_{h}^{\infty}:=H^{\infty}\left(\Sigma_{h}, X\right) \cap C_{b}\left(\overline{\Sigma_{h}}, X\right),
$$

that is, the space of bounded holomorphic functions continuous to the boundary equipped with sup-norm, and

$$
Y_{h}^{q}:=H^{\infty}\left(\Sigma_{h}, X\right) \cap L^{q}\left(\Sigma_{h}, X\right),
$$

that is, the space of holomorphic functions with the norm

$$
\|f\|_{q}:=\left(\iint_{\Sigma_{h}}\|f(x+i y)\|^{q} d x d y\right)^{1 / q}<+\infty .
$$

The left translation semigroup on these spaces is defined by

$$
(S(t) f)(z):=f(t+z)
$$

It is easy to show that

$$
D f=f^{\prime}, \quad D(D)=\left\{f \in Y^{q}: f^{\prime} \in Y^{q}\right\}
$$

is the generator of $S$ (where $1 \leq q \leq+\infty)$ ). Moreover, $S$ is differentiable since for every $t>0$ we have $S(T) f \in D(D)$. In fact, the Cauchy formula yields

$$
\left\|f^{\prime}(t+z)\right\| \leq \frac{1}{2 \pi} \int_{\gamma} \frac{\|f(w)\|}{r^{2}}|d w|
$$

and the diameter $r$ of the circle $\gamma$ centered in $t+z$ is independent of $z$ since the distance of the shifted area $\Sigma_{h}+t$ from the boundary of $\Sigma_{h}$ is positive. Then

$$
\left\|f^{\prime}(t+z)\right\| \leq \frac{1}{r} \sup \{\|f(w)\|: w \in \gamma\}
$$

and we have proved the differentiability on $Y^{\infty}$. The $L^{q}$ estimate follows from (2.1) by Fubini's theorem and Hölder's inequality.

However, the semigroup $S$ is more than just differentiable. It has an analytic extension to $\Sigma_{h}$. In fact, we can define

$$
\left.(S(z) f)(w):=f(z+w) \text { for } z \in \Sigma_{h} \cup\{0\}, w \in \Sigma_{h} \text { (or } w \in \overline{\Sigma_{h}} \text { if } q=+\infty\right)
$$

and this extension has the complex derivative. If $h^{\prime}(0)>0$, then there exists a sector $\Sigma_{\varphi} \subset \Sigma_{h}$ and the semigroup $S$ is analytic. If $h^{\prime}(0)=0$, then there is no sector (with the vertex in zero) contained in $\Sigma_{h}$. Hence, in this case $S$ is not an analytic semigroup. 


\section{On bounded perturbations of differentiable semigroups}

In the next section we show that solutions of (1.1) are $C^{\infty}$ under appropriate assumptions on $A$ and $B$. Our main tool will be perturbation theory. Therefore, we devote the present section to bounded perturbations of generators of differentiable semigroups.

If $A$ is the generator of a $C_{0}$-semigroup (respectively norm-continuous semigroup, analytic semigroup) and $B$ is bounded, then $A+B$ is again a generator of a $C_{0^{-}}$ semigroup (respectively norm-continuous semigroup, analytic semigroup). These properties are preserved under bounded perturbations. Unfortunately, the same is not true for differentiable semigroups. Renardy in [11] gave an example of a generator of a differentiable semigroup and its perturbation which is not differentiable. On the other hand, Doytchinov et al. [5] proved that all bounded perturbations of a differentiable semigroup $T$ are differentiable if the semigroup $T$ satisfies

$$
\limsup _{t \rightarrow 0} \frac{t \cdot \log \left(\left\|T^{\prime}(t)\right\|\right)}{\log (1 / t)}=L<+\infty .
$$

Pazy [9] gave conditions on the resolvent of the generator $A$. In fact, if for every $\beta>0$ there exists $c \in \mathbb{R}$ such that

$$
D_{\beta, c} \subset \rho(A) \quad \text { and } \quad \lim _{|\operatorname{Im} \lambda| \rightarrow \infty, \lambda \in D_{\beta, c}}\|R(\lambda, A)\|=0,
$$

then every bounded perturbation of $A$ generates a differentiable semigroup. Here

$$
D_{\beta, c}:=\{\lambda \in \mathbb{C}: \operatorname{Re} \lambda \geq c-\beta \log |\lambda|\} .
$$

Iley showed in [7] that the condition (3.2) is also necessary. A consequence of this fact is that if $A$ satisfies (3.2) then every bounded perturbation of $A$ satisfies (3.2) as well. We show that semigroups with analytic extensions to $\Sigma_{h}$ satisfy (3.2).

LEMMA 3.1. Let $f \in C\left(\mathbb{R}_{+}, X\right)$ and its restriction to $(T, \infty)$ have a bounded analytic extension to $T+\Sigma_{\varphi}, \varphi \in(0, \pi / 2]$. Then the Laplace transform $\widehat{f}(\lambda)$ exists for all $\lambda$ with $|\arg \lambda|<\pi / 2+\varphi$ and there exists $M>0$ such that

$$
\|\widehat{f}(\lambda)\| \leq \frac{M}{|\lambda|}\left(1+\left|e^{-\lambda T}\right|\right)
$$

holds for all $\lambda \in \Sigma_{\pi / 2+\varphi}$.

Proof. Since $g(s):=f(T+s)$ is bounded and analytic in $\Sigma_{\varphi}$, there exists $\widehat{g}(\lambda)$ for all $\lambda \in \Sigma_{\pi / 2+\varphi}$ and

$$
\|\widehat{g}(\lambda)\| \leq \frac{\tilde{M}}{|\lambda|}
$$


holds on subsectors of $\Sigma_{\pi / 2+\varphi}$. It follows from boundedness of $f$ that $M$ is independent of subsectors. We have

$$
\begin{aligned}
\widehat{g}(\lambda) & =\int_{0}^{+\infty} e^{-\lambda s} g(s) d s=e^{\lambda T} \int_{0}^{+\infty} e^{-\lambda(s+T)} f(s+T) d s \\
& =e^{\lambda T} \int_{T}^{+\infty} e^{-\lambda s} f(s) d s=e^{\lambda T}\left(\widehat{f}(\lambda)-\int_{0}^{T} e^{-\lambda s} f(s) d s\right) .
\end{aligned}
$$

It follows that

$$
\|\widehat{f}(\lambda)\| \leq e^{-\lambda T} \cdot \frac{\tilde{M}}{|\lambda|}+\sup _{t \in[0, T]}\|f(t)\|\left|\frac{e^{-\lambda T}-1}{-\lambda}\right| \leq \frac{M}{|\lambda|}\left(1+\left|e^{-\lambda T}\right|\right) .
$$

The following theorem is an easy consequence of Lemma 3.1.

THEOREM 3.2. Let $T$ be a $C_{0}$-semigroup with a bounded analytic extension to $\Sigma_{h}$ for an admissible function $h$. Let $A$ be the generator of $T$. Denote $\theta=\lim _{x \rightarrow \infty} \operatorname{arctg}\left(\varphi^{\prime}(x)\right)$. Then $\Sigma_{\pi / 2+\theta} \subset \rho(A)$ and there exist $c_{\alpha}, M>0$ such that

$$
\|R(\lambda, A)\| \leq \frac{M}{|\lambda|}\left(1+\left|e^{-\lambda c_{\alpha}}\right|\right)
$$

for all $\lambda \in \Sigma_{\pi / 2+\alpha}, \alpha<\theta$. Moreover, $c_{\alpha}$ can be taken in such a way that $c_{\alpha} \rightarrow 0$ for $\alpha \rightarrow 0$.

THEOREM 3.3. Let $T$ be a $C_{0}$-semigroup with a bounded analytic extension to $\Sigma_{h}$ for an admissible function $h$. Let $A$ be the generator of T. Then (3.2) holds.

Proof. Let $\beta>0$. Take $c \in \mathbb{R}$ such that $D_{\beta, c} \subset \rho(A)$ and $\lambda_{n} \in D_{\beta, c}$ such that $\left|\operatorname{Im} \lambda_{n}\right| \rightarrow \infty$. Then $\left|\arg \lambda_{n}\right| \rightarrow \pi / 2$. It follows that

$$
\begin{aligned}
\left\|R\left(\lambda_{n}, A\right)\right\| & \leq \frac{M}{\left|\operatorname{Im} \lambda_{n}\right|}\left(1+e^{-\operatorname{Re} \lambda_{n} c_{n}}\right) \leq \frac{M+M e^{c_{n}\left(\beta \log \left|\operatorname{Im} \lambda_{n}\right|-c\right)}}{\left|\operatorname{Im} \lambda_{n}\right|} \\
& \leq \frac{M+M e^{-c_{n} c}\left|\operatorname{Im} \lambda_{n}\right|^{c_{n} \beta}}{\left|\operatorname{Im} \lambda_{n}\right|} \rightarrow 0
\end{aligned}
$$

since $c_{n} \beta<1$ for large $n$.

Condition (3.1) of Doytchinov et al. [5] is stronger than (3.2). Since the growth of $T^{\prime}$ is given by the shape of $\Sigma_{h}$, we can only expect (3.1) for not very sharp cusps in zero.

THEOREM 3.4. Let $A$ be the generator of a $C_{0}$-semigroup $T$ with a bounded analytic extension to $\Sigma_{h}$ for an admissible function $h$. Let

$$
\limsup _{t \rightarrow 0} \frac{t \cdot \log (1 / h(t))}{\log (1 / t)}=L<+\infty .
$$

Then T satisfies (3.1). 
Proof. From the Cauchy formula we obtain

$$
\left\|T^{\prime}(t)\right\| \leq \frac{1}{2 \pi} \int_{0}^{2 \pi} \frac{\left\|T\left(t+r e^{i \alpha}\right)\right\|}{r^{2}} \cdot r d \alpha \leq \frac{M}{r},
$$

where the disc of radius $r$ centered in $t$ is a subset of $\Sigma_{h}$. So, $r$ depends on $t$ and for small $t$ we can take $r=h(t) / 2$; hence

$$
\left\|T^{\prime}(t)\right\| \leq \frac{2 M}{h(t)}
$$

and (3.1) follows.

Since not only (3.7) but also the opposite inequality holds, we have examples of $T$ with differentaible bounded perturbations and with arbitrarily fast growth of $\left\|T^{\prime}\right\|$ at zero.

\section{Differentiable solutions of integrodifferential equations}

In this section we show that the semigroup $\mathcal{T}$ generated by $\mathcal{A}$ (defined in the introduction) is differentiable if $B$ is in an appropriate space. First we consider the case of $Y^{\infty}$ and then the case of $Y^{q}$.

THEOREM 4.1. Let $A$ be the generator of a differentiable semigroup $T$ satisfying (3.2). Let $B, B^{\prime} \in Y^{\infty}\left(\Sigma_{h}, B(D(A), X)\right)$, where $h$ is an admissible function. Then $\mathcal{A}$ generates a differentiable semigroup on $X \times Y^{\infty}\left(\Sigma_{h}, X\right)$.

Proof. Since $A$ and the operator $D$ on $Y^{\infty}\left(\Sigma_{h}, X\right)$ satisfy (3.2), the operator

$$
\begin{gathered}
\mathcal{A}_{00}:=\left(\begin{array}{ll}
A & 0 \\
0 & D
\end{array}\right), \quad D\left(\mathcal{A}_{00}\right):=D(A) \times D(D) \quad \text { with } \\
R\left(\lambda, \mathcal{A}_{00}\right)=\left(\begin{array}{cc}
R(\lambda, A) & 0 \\
0 & R(\lambda, D)
\end{array}\right)
\end{gathered}
$$

satisfies (3.2) as well. Since

$$
\mathcal{B}_{1}:=\left(\begin{array}{cc}
0 & \delta_{0} \\
0 & 0
\end{array}\right)
$$

is bounded, operator $\mathcal{A}_{0}:=\mathcal{A}_{00}+\mathcal{B}_{1}$ generates a differentiable semigroup and satisfies (3.2) by Iley [7].

Since

$$
\mathcal{B}_{2}:=\left(\begin{array}{ll}
0 & 0 \\
B & 0
\end{array}\right)
$$

is a bounded operator on $D\left(\mathcal{A}_{0}\right)$ with the graph norm, the operator $\mathcal{A}:=\mathcal{A}_{0}+\mathcal{B}$ generates a $C_{0}$-semigroup by [4] (see [6, Corollary III.1.5]). The resolvent operator of $\left.\mathcal{A}_{0}\right|_{D\left(\mathcal{A}_{0}\right)}$ is similar to the resolvent operator of $\mathcal{A}_{0}$, according to [6, Section II.5a]. Hence, the restricted resolvent operator satisfies (3.2). It follows that the restriction of the semigroup generated by $\mathcal{A}$ is differentiable. Since the semigroup generated by $\mathcal{A}$ is similar to its differentiable restriction, it is differentiable too. 
The case $Y^{q}$ is more complicated, since $\delta_{0}$ is not a bounded operator from $Y^{q}\left(\Sigma_{h}, X\right) \rightarrow X$. Therefore, it is not true that $\mathcal{A}_{0}$ generates a differentiable semigroup and satisfies (3.2) for all $q$ and $h$. We shall prove the following theorem.

THEOREM 4.2. Let A satisfy (3.2). Let h be an admissible function satisfying

$$
\int_{0}^{1}|h(x)|^{1-s} d x<+\infty
$$

for some $s>1$. Let $B, B^{\prime} \in Y^{q}\left(\Sigma_{h}, B(D(A), X)\right)$ for some $q \geq s /(s-1)$. Then $\mathcal{A}$ generates a differentiable semigroup on $X \times Y^{q}\left(\Sigma_{h}, X\right)$.

In fact, $s<2$ since $h(x) \geq c x$ for some $c>0$ in the neighborhood of 0 . It follows that $q>2$. Condition (4.1) means that the cusp of $\Sigma_{h}$ in zero is polynomial (not exponential).

If $\mathcal{A}_{0}$ satisfies (3.2), then the rest of the proof is the same as in Theorem 4.1. We first show that $\mathcal{A}_{0}$ generates a $C_{0}$-semigroup, then we show that $R\left(\lambda, \mathcal{A}_{0}\right)$ satisfies (3.2).

Since $A$ and $D$ generate $C_{0}$-semigroups $T$ and $S$ on $X$ and $Y_{h}^{q}$, respectively, then $\mathcal{A}_{0}$ generates a $C_{0}$-semigroup if and only if

$$
R(t) f:=\int_{0}^{t} T(t-s) \delta S(s) f d s=\int_{0}^{t} T(t-s) f(s) d s
$$

extends to a bounded operator from $Y_{h}^{q}$ to $X$ satisfying $\lim \sup _{t \rightarrow 0}\|R(t)\|<\infty$. The semigroup generated by $\mathcal{A}_{0}$ is then given by

$$
\mathcal{T}_{0}(t):=\left(\begin{array}{cc}
T(t) & R(t) \\
0 & S(t)
\end{array}\right)
$$

This result is due to Nagel [8, Proposition 3.1].

Let us start with the following lemma, which will be of help for proving boundedness of $R(t)$ as well as (3.2).

LEMMA 4.3. Let $h$ be an admissible function and $s>1$ be such that (4.1) holds. Then for every $q \geq s /(s-1)$ and every $R>0$ there exists $C>0$ such that

$$
\int_{0}^{R}\|f(t)\| d t \leq C\|f\|_{q}
$$

holds for every $f \in Y_{h}^{q}$.

PROOF. We estimate the value of $\|f(r)\|$ using the Cauchy formula. The integration path will consist of two circle segments. Let $\gamma_{1}(t):=r+c \cdot h(r)-h(r) e^{i t}$, $t \in[-\alpha, \alpha]$ and $\gamma_{2}(t):=r-c \cdot h(r)+h(r) e^{i t}, \quad t \in[-\alpha, \alpha]$ with $c<1$ and $\alpha:=\operatorname{arc} \cos c$. Since $h^{\prime}(r)$ is bounded on $[0, R], \gamma:=\gamma_{1}+\gamma_{2}$ is contained in $\Sigma_{h}$ for every $r$ if $1-c$ is small enough. In particular, we need $h^{\prime}(r)<\operatorname{cotg} \alpha$. 
We have

$$
\begin{aligned}
\|f(r)\|= & \left\|\frac{1}{2 \pi i} \int_{\gamma} \frac{f(z)}{z-r} d z\right\| \leq \frac{1}{2 \pi} \int_{-\alpha}^{\alpha} \frac{\left\|f\left(r+\operatorname{ch}(r)-h(r) e^{i t}\right)\right\|}{\operatorname{ch}(r)}\left\|h(r) i e^{i t}\right\| d t \\
& +\frac{1}{2 \pi} \int_{-\alpha}^{\alpha} \frac{\left\|f\left(r-\operatorname{ch}(r)+h(r) e^{i t}\right)\right\|}{\operatorname{ch}(r)}\left\|h(r) i e^{i t}\right\| d t .
\end{aligned}
$$

Hence,

$$
\begin{aligned}
\int_{\rho}^{R}\|f(r)\| d r \leq & C \iint_{M}\left\|f\left(r+\operatorname{ch}(r)-h(r) e^{i t}\right)\right\| d r d t \\
& +C \iint_{M}\left\|f\left(r-\operatorname{ch}(r)+h(r) e^{i t}\right)\right\| d r d t
\end{aligned}
$$

for $M:=(\rho, R) \times(-\alpha, \alpha)$. We shall estimate the first integral, and the second one can be estimated in a similar way. A change of variables

$$
\begin{aligned}
\psi(r, t) & :=(r+c h(r)-h(r) \cos t, h(r) \sin t), \\
\left|J_{\psi}\right| & =\left|h(r)\left(\cos t+c^{\prime}(r) \cos t-h^{\prime}(r)\right)\right|
\end{aligned}
$$

yields

$$
\iint_{M}\left\|f\left(r+\operatorname{ch}(r)-h(r) e^{i t}\right)\right\| d r d t \leq \iint_{M^{\prime}} \frac{\|f(x+i y)\|}{c_{1} h(x)} d x d y
$$

with $M^{\prime}=\psi(M)$. In fact, from $h^{\prime}(r)<\operatorname{cotg} \alpha$ we obtain

$$
\left|J_{\psi}\right| \geq|h(r)| c\left(1-\sqrt{1-c^{2}}\right)
$$

Since $|h(r)| \geq \widetilde{c}|h(x)|$, there exists a constant $c_{1}$ such that (4.4) holds. Also

$$
M^{\prime} \subset M^{\prime \prime}:=\left\{x+i y \in \mathbb{C}:|y|<c^{\prime} h(x), x \in(0, R+\delta)\right\}
$$

holds for an appropriate $0<c^{\prime}<1$. By this inclusion and Hölder's inequality, (4.4) is estimated by

$$
\frac{1}{c_{1}}\left(\int_{M^{\prime \prime}}\|f(x+i y)\|^{s^{\prime}} d x d y\right)^{1 / s^{\prime}}\left(\int_{M^{\prime \prime}} \frac{1}{h(x)^{s}} d x d y\right)^{1 / s},
$$

where $s^{\prime}=s /(s-1)$. Since $M^{\prime \prime}$ is bounded, we have $L^{q} \hookrightarrow L^{s^{\prime}}$ for $q>s^{\prime}$. The second integral in (4.5) is equal to

$$
\left(\int_{0}^{R+\delta} \frac{c^{\prime} h(x)}{h(x)^{s}} d x\right)^{1 / s}=\widetilde{C}<+\infty
$$

by the assumptions of this lemma. This proves (4.3). 
If the estimate (4.3) holds, we can write

$$
\|R(t) f\| \leq \sup _{0 \leq s \leq t}\|T(s)\| \int_{0}^{t}\|f(s)\| d s \leq C\|f\|_{q} .
$$

Hence, $R(\cdot)$ satisfies both of the properties mentioned before Lemma 4.3 and $\mathcal{A}_{0}$ is the generator of a $C_{0}$-semigroup. We have proved the following theorem.

THEOREM 4.4. Let $h$ be an admissible function and $s>1$ be such that (4.1) holds. Let $A$ be the generator of a $C_{0}$-semigroup $T$ on $X$. Then $\mathcal{A}_{0}$ generates a $C_{0}$-semigroup on $X \times L^{q}\left(\Sigma_{h}, X\right)$ for every $q \geq s /(s-1)$.

In the following two lemmas we show that $\mathcal{A}_{0}$ satisfies (3.2). In fact, the first lemma says that the left shift semigroup satisfies a stronger growth condition than that by Doytchinov et al. [5]. The second lemma shows that if this condition holds, then the operator $\mathcal{A}_{0}$ on the product space satisfies (3.2).

LEMMA 4.5. Let $s>1$ and $h$ be an admissible function satisfying (4.1). Then $h$ satisfies

$$
\limsup _{t \rightarrow 0+} t \log (1 / h(t))=0 .
$$

PROOF. Assume for contradiction that there exist $\varepsilon>0$ and a sequence $t_{n} \rightarrow 0$ such that

$$
t_{n} \log \left(1 / h\left(t_{n}\right)\right)>\varepsilon
$$

Hence,

$$
\frac{1}{h\left(t_{n}\right)} \geq e^{\varepsilon / t_{n}}
$$

Since $h$ is increasing,

$$
\int_{0}^{1} \frac{1}{h(t)^{s-1}} d t \geq \sum_{n=k}^{\infty} e^{\varepsilon(s-1) / t_{n}}\left(t_{n}-t_{n+1}\right) .
$$

Since we can choose the sequence such that $t_{n+1}<t_{n} / 2$, the right-hand side of (4.7) is greater than

$$
\frac{1}{2} \sum_{n=k}^{\infty} e^{\varepsilon(s-1) / t_{n}+\log t_{n}}=+\infty .
$$

This means that (4.1) does not hold.

LEMMA 4.6. Let $s>1$ and $h$ be an admissible function satisfying (4.1). Then $\mathcal{A}_{0}$ satisfies (3.2).

PROOF. Since the resolvent of $\mathcal{A}_{0}$ is equal to

$$
R\left(\lambda, \mathcal{A}_{0}\right)=\left(\begin{array}{cc}
R(\lambda, A) & R(\lambda, A) \delta_{0} R(\lambda, D) \\
0 & R(\lambda, D)
\end{array}\right),
$$


and $\|R(\lambda, A)\|,\|R(\lambda, D)\| \rightarrow 0$, it is sufficient to show that $\left\|\delta_{0} R(\lambda, D)\right\|$ is bounded on $D_{\beta, c} \cap\{\lambda>K\}$.

We have the following Sobolev-type imbedding. There exists $L>0$ such that

$$
\sup _{z \in \Omega}\|f(z)\| \leq L\left(\|f\|_{Y^{q}}+\left\|f^{\prime}\right\|_{Y^{q}}\right)
$$

for all $f \in D(D)$. In fact, inequality (4.8) follows from the Sobolev imbedding $W^{1, q} \hookrightarrow C_{B}$ on $\Omega \cap\{z:|z|>\varepsilon\}$ since this set has a nice boundary and $q>2$ according to the remark below Theorem 4.2. For $z$ near the cusp $(|z| \leq \varepsilon)$ we have

$$
\|f(z)\| \leq\|f(R)\|+\left\|\int_{z}^{R} f^{\prime}(t) d t\right\| \leq\|f(R)\|+C\left\|f^{\prime}\right\|_{q}
$$

by Lemma 4.3 if $z \in \mathbb{R}$. Here $\|f(R)\|$ is estimated by $W^{1, q}$-norm since $|R|>\varepsilon$. If $z \notin \mathbb{R}$ we can write

$$
\|f(z)\| \leq\|f(i \operatorname{Im} z+R)\|+\left\|\int_{\operatorname{Re} z}^{R} f^{\prime}(i \operatorname{Im} z+t) d t\right\| \leq\|f(R)\|+C\left\|f^{\prime}\right\|_{q},
$$

where the last estimate follows in a similar way as in Lemma 4.3.

From (4.8) we have

$$
\begin{aligned}
\left\|\delta_{0} R(\lambda, D)\right\| & \leq L(\|D R(\lambda, D)\|+\|R(\lambda, D)\|)=L(\|\lambda R(\lambda, D)-I\|+\|R(\lambda, D)\|) \\
& \leq L+L|\lambda|\|R(\lambda, D)\|+L\|R(\lambda, D)\| .
\end{aligned}
$$

By Theorem 3.2 we have

$$
\|R(\lambda, D)\| \leq \frac{M}{|\lambda|}\left(1+e^{-\operatorname{Re} \lambda T_{\alpha}}\right)
$$

for $\lambda \in \Sigma_{\pi / 2+\alpha}$. So, it is sufficient to show that $-\operatorname{Re} \lambda T_{\alpha}$ is bounded from above for $\lambda \rightarrow \infty, \lambda \in D_{\beta, c}$.

Take $K>0$ large enough and $\lambda \in D_{\beta, c},|\lambda|>K$. Then $-\operatorname{Re} \lambda \leq \beta \log K-c$. Moreover, we have

$$
\frac{-\operatorname{Re} \lambda}{|\operatorname{Im} \lambda|} \leq \frac{\beta \log K-c}{K}=: \operatorname{tg} \alpha .
$$

We show that $(\beta \log K-c) T_{\alpha}$ remains bounded as $K \rightarrow+\infty$.

It follows from Lemma 3.1 that $T_{\alpha}$ should be such that $T_{\alpha}+\Sigma_{\alpha} \subset \Sigma_{h}$. Hence, we can take

$$
T_{\alpha}:=\inf \left\{t>0: h^{\prime}(t) \geq \operatorname{tg} \alpha\right\} .
$$

From (4.6) we have $t \log (1 / h(t)) \leq \varepsilon$ for all $t$ small enough. Hence,

$$
h(t) \geq e^{-\varepsilon / t} .
$$


Since $h^{\prime}$ is nondecreasing, we obtain $h^{\prime}(t) \geq\left(\left(e^{-\varepsilon / t}\right) / t\right)=e^{-\varepsilon / t-\log t} \geq e^{-(\varepsilon+1) / t}$ if $t$ is small enough. Hence, taking $z=e^{-(\varepsilon+1) / t}$ we obtain

$$
h^{\prime}\left(-\frac{\varepsilon+1}{\log z}\right) \geq z, \quad \text { for all } z \text { small enough. }
$$

For $K$ large $\operatorname{tg} \alpha$ is small (see (4.9)). So, we can take $z:=\operatorname{tg} \alpha$ in (4.11) and obtain

$$
T_{\alpha} \leq-\frac{\varepsilon+1}{\ln \operatorname{tg} \alpha}
$$

by (4.10). Now (4.8) and (4.9) yield

$$
T_{\alpha}(\beta \log K-c) \leq \frac{(\varepsilon+1)(\beta \log K-c)}{\log K-\log (\beta \log K-c)-1} \rightarrow \beta(\varepsilon+1)
$$

as $K \rightarrow+\infty$.

This lemma completes the proof of Theorem 4.2.

A special case of an admissible function $h$ is $h(t)=\operatorname{tg}(\varphi) \cdot t$. In this case, $\Sigma_{h}=\Sigma_{\varphi}$ is a sector and the shift semigroup on this sector is even analytic. However, if $A$ is not a generator of an analytic semigroup, one cannot expect that the semigroup $\mathcal{T}$ is analytic. In this case, the conditions on $h$ and $q$ become more simple.

COROLlary 4.7. Let $A$ be the generator of a differentiable semigroup $T$ satisfying (3.2) and $0<\varphi \leq \pi / 2$. Let $B, B^{\prime} \in Y^{q}\left(\Sigma_{\varphi}, B(D(A), X)\right)$ for some $q>2$ or $q=\infty$. Then $\mathcal{A}$ generates a differentiable semigroup on $X \times Y^{q}\left(\Sigma_{\varphi}, X\right)$.

Our results are neither weaker nor stronger than the results in [10]. In fact, our result does not apply to kernels which are unbounded in the neighborhood of zero. On the other hand, it applies to some kernels that are not $k$-regular for all $k \in \mathbb{N}$. Let $a$ be any scalar function such that $a^{\prime}, a^{\prime \prime} \in Y_{h}^{q}$ and $a(t)>0$ for $t>0$ (for example $a(z)=1 /(z+1))$. Then $a-c$ is not $k$-regular for some constant $c>0$, since $\widehat{a}(\lambda)=0$ for some $\lambda>0$. Hence, [10, Theorem 3.1] does not apply to

$$
u(t)=\int_{0}^{t} a(t-s) \tilde{A} u(s) d s+x .
$$

However, differentiating (4.12) we obtain our Equation (1.1) with $A=a(0) \widetilde{A}$ and $B(s)=a^{\prime}(s) \widetilde{A}$ and Theorems 4.1 and 4.2 can be applied.

\section{References}

[1] T. Barta, 'Analytic solutions for integrodifferential equations via semigroups', Semigroup Forum 76 (2008), 142-148.

[2] - 'On $R$-sectorial derivatives on Bergman spaces', Bull. Aust. Math. Soc. 77 (2008), 305-313.

[3] C. J. K. Batty, Differentiability of Perturbed Semigroups and Delay Semigroups, Banach Center Publications, 75 (Polish Academy of Science, Warsaw, 2007). 
[4] W. Desch and W. Schappacher, 'On relatively bounded perturbations of linear $C_{0}$ semigroups', Ann. Scuola Norm. Sup. Pisa Cl. Sci. 11 (1984), 327-341.

[5] B. Doytchinov, W. J. Hrusa and S. J. Watson, 'On perturbations of differentiable semigroups', Semigroup Forum 54 (1997), 100-111.

[6] K. J. Engel and R. Nagel, One-parameter Semigroups for Linear Evolution Equations (Springer, New York, 2000).

[7] P. Iley, 'Perturbations of differentiable semigroups', J. Evol. Eq. 7 (2007), 765-781.

[8] R. Nagel, 'Towards a 'matrix theory' for unbounded operator matrices', Math. Z. 201 (1989), $57-68$.

[9] A. Pazy, 'On the differentiability and compactness of semi-groups of linear operators', J. Math. Mech. 17 (1968), 1131-1141.

[10] J. Pruss, Evolutionary integral equations and applications (Birkhauser, Basel, 1993).

[11] M. Renardy, 'On the stability of differentiability of semigroups', Semigroup Forum 51 (1995), 343-346.

TOMÁŠ BÁRTA, Faculty of Mathematics and Physics,

Department of Mathematical Analysis, Charles University,

Prague, Sokolovska 83,180 00 Prague 8, Czech Republic

e-mail: barta@karlin.mff.cuni.cz 